\title{
Functional Coupling between the Prefrontal Cortex and Dopamine Neurons in the Ventral Tegmental Area
}

\author{
Ming Gao, ${ }^{1}$ Chang-Liang Liu, ${ }^{1}$ Shen Yang, ${ }^{1}$ Guo-Zhang Jin, ${ }^{1}$ Benjamin S. Bunney, ${ }^{2}$ and Wei-Xing Shi ${ }^{1,2}$ \\ ${ }^{1}$ Department of Pharmacology, Shanghai Institute of Materia Medica, Shanghai Institutes of Biological Sciences, Chinese Academy of Sciences, Shanghai \\ 201203, China, and 2Neuropsychopharmacological Research Unit, Department of Psychiatry, Yale University School of Medicine, New Haven, Connecticut \\ 06511
}

\begin{abstract}
Stimulation of the prefrontal cortex (PFC) has been shown to have an excitatory influence on dopamine (DA) neurons. We report here that, under nonstimulated conditions, the activity of DA neurons in the ventral tegmental area (VTA) also covaries, on a subsecond timescale, with the activity of PFC cells. Thus, in $67 \%$ of VTA DA neurons recorded in chloral hydrate-anesthetized rats, the firing of the cell displayed a slow oscillation (SO) that was highly coherent with the activity of PFC neurons. The SO was suppressed by transections immediately caudal to the PFC or by intra-PFC infusion of tetrodotoxin, suggesting that it depends on inputs derived from the PFC. Unexpectedly, the SO in most VTA DA neurons was reversed in phase relative to PFC cell activity, suggesting that at least part of PFC information is transferred to DA neurons indirectly through inhibitory relay neurons. These results, together with those reported previously, suggest that the PFC can act through multiple pathways to exert both excitatory and inhibitory influences on DA neurons. The observed functional coupling between DA and PFC neurons further suggests that these pathways not only allow a bidirectional control of DA neurons by the PFC, but also enable action potential-dependent DA release to be coordinated, on a subsecond timescale, with glutamate release from PFC terminals. Further understanding of this coordinated activity may provide important new insights into brain functions and disorders thought to involve both VTA DA and PFC neurons.
\end{abstract}

Key words: prefrontal cortex; ventral tegmental area; dopamine neuron; firing pattern; slow oscillation; bursting

\section{Introduction}

The prefrontal cortex (PFC) is a key structure for executive functions of the brain (Miller and Cohen, 2001; Miller et al., 2002) and has been shown to regulate the firing pattern of dopamine (DA) neurons in the ventral tegmental area (VTA). Thus, PFC stimulation increases burst firing in DA neurons, whereas PFC inactivation produces the opposite effect (Gariano and Groves, 1988; Svensson and Tung, 1989; Murase et al., 1993; Overton et al., 1996; Tong et al., 1996). Consistent with the notion that bursting is more effective than single-spike firing in inducing DA release (Gonon, 1988), PFC stimulation also increases extracellular DA levels in the nucleus accumbens (NAc) (Murase et al., 1993; Taber and Fibiger, 1995; Taber et al., 1995; Karreman and Moghaddam, 1996; You et al., 1998). This increase is blocked by glutamate antagonists locally infused into the VTA, but not into the NAc, suggesting that the effect is secondary to glutamate release in the VTA and subsequent activation of DA neurons projecting to the NAc. However, because PFC glutamate terminals

\footnotetext{
Received Dec. 12, 2006; revised March 25, 2007; accepted April 16, 2007.

This work was supported by Chinese National Basic Research Program Grant 973-2003CB515400, Chinese National Natural Science Foundation Grant 30572168, National Institute on Drug Abuse Grant DA12944, a National Alliance for Research on Schizophrenia and Depression (NARSAD) Independent Investigator Award (W.-X.S.), and a NARSAD Distinguished Investigator Award (B.S.B.)

Correspondence should be addressed to Dr. Wei-Xing Shi, Neuropsychopharmacological Research Unit, Yale University School of Medicine, 300 George Street, Room 8300C, New Haven, CT 06511. E-mail: wei-xing.shi@yale.edu.

D01:10.1523/JNEUROSC1.5347-06.2007

Copyright $\odot 2007$ Society for Neuroscience $\quad$ 0270-6474/07/275414-08\$15.00/0
}

do not innervate NAc-projecting DA neurons directly (Carr and Sesack, 2000a; Sesack et al., 2003), the increase in DA release induced by PFC stimulation is likely to be indirectly mediated by PFC projections to other brain areas, which, in turn, provide direct glutamate inputs to DA neurons. Consistent with this possibility, the bursts in DA neurons induced by PFC stimulation often show a long latency (150-223 ms), which is well beyond what normally expected for monosynaptic events (Gariano and Groves, 1988; Overton and Clark, 1997).

We showed previously that rhythmic bursting observed in many VTA DA neurons can be described as a slow oscillation (SO) in firing rate (Shi, 2005). The frequency of the SO corresponds to the frequency of burst-like events and its amplitude is correlated with both the number and frequency of spikes within each event. Both can be determined using spectral analysis. The presence of the SO in DA neurons provides a unique opportunity to use a variety of techniques to study how DA cell activity is correlated with and regulated by activities in other brain areas. Given the role of the PFC in DA cell bursting and the similarity between bursting and the SO, this study, by using cross-spectral and coherence analysis, examines whether the SO in DA neurons encodes information from the PFC. A possible role of the PFC in the $\mathrm{SO}$ is also suggested by a previous study showing that local field potentials (LFPs) recorded in the VTA also exhibit an SO, which is highly synchronized with the activity of PFC neurons (Peters et al., 2004). However, because DA neurons are interspersed with non-DA neurons in the VTA, it is uncertain whether 
VTA LFPs reflect the activity of DA or non-DA neurons. Thus, the second goal of this study was to determine how firing patterns of individual DA and non-DA neurons are related to LFPs recorded in the VTA.

\section{Materials and Methods}

Electrophysiological recordings. All procedures were in compliance with the Guide for the Care and Use of Laboratory Animals (National Research Council, People's Republic of China, 1996). Male Sprague Dawley rats, weighing between 250 and $300 \mathrm{~g}$, were anesthetized with chloral hydrate $(400 \mathrm{mg} / \mathrm{kg}$, i.p., with supplemental doses administered via a lateral tail vein) and mounted in a stereotaxic apparatus. All pressure points and incision sites were infiltrated with the local anesthetic bupivacaine hydrochloride (0.25\%; Harvest Pharmaceutical, Shanghai, China). Singleunit activities were recorded extracellularly using techniques similar to those described previously (Shi et al., 2000, 2004). VTA DA and non-DA neurons were identified by well established electrophysiological criteria (Bunney et al., 1973; Grace and Bunney, 1983; Ungless et al., 2004). In most experiments, multiple cells were recorded from each animal either randomly or using the "cells per track" technique (Bunney and Grace, 1978; Chiodo and Bunney, 1983). When the latter technique was used, 12 electrode tracks were made in the right side of the VTA [from lambda, anteroposterior (AP), 2.7-3.3; mediolateral (ML), 0.5-0.9; dorsoventral (DV), $6.5-8.5 \mathrm{~mm}$ ]. Each track was separated by $200 \mu \mathrm{m}$. To simultaneously record VTA LFPs, signals were fed into two bandpass filters, one for single-unit detection (from $80 \mathrm{~Hz}$ to $10 \mathrm{kHz}$ ) and one for spike-free LFP recording $(0-30 \mathrm{~Hz})$. PFC LFPs were recorded through a separate electrode inserted in the PFC (from bregma, AP, 3.2; ML, 0.7; DV, 3.5 $\mathrm{mm}$ ). In a subgroup of rats, single-unit recordings were made from PFC neurons (from bregma, AP, 2.7-3.3; ML, 0.6-0.8; DV, 2.5-4.0 mm). Throughout the experiment, body temperature was maintained at 36$38^{\circ} \mathrm{C}$ with a homeothermic blanket system (Harvard Apparatus, Holliston, MA). At the end of experiments, recording sites were marked by ejection of pontamine sky blue and examined using standard histology methods.

PFC transection and TTX infusion. To test whether PFC inputs are involved in the generation of the SO in DA neurons, bilateral transections were made immediately caudal to the PFC to interrupt PFC inputs to DA neurons. A slit was drilled in the skull $2.0 \mathrm{~mm}$ anterior to bregma. The sharp pointed tip of a 3-mm-wide scalp blade was lowered to the base of skull and passed along the slit to completely interrupt connections between the PFC and the rest of the brain. Recordings commenced $30 \mathrm{~min}$ after the transection.

In a second group of rats, the PFC was inactivated by local infusion of TTX (Fisheries Science and Technology Development, Hebei, China). Silica capillary tubing (outer diameter, $160 \mu \mathrm{m}$; inner diameter, $100 \mu \mathrm{m}$ ) was filled with TTX (10 ng/ $\mu \mathrm{l}$ in $0.9 \%$ saline $)$ and inserted into the medial PFC (from bregma, AP, 3.0; ML, 0.7; DV, $3.5 \mathrm{~mm}$ ). After a stable baseline recording, TTX was infused at $0.75 \mu \mathrm{l} / \mathrm{min}$ for $2 \mathrm{~min}$ (controlled by a syringe pump; Harvard Apparatus). Effects of TTX infusion were determined by comparing recordings from the same cell before and after the infusion. Only one cell was studied in each rat. In separate groups of animals, saline $(1.5 \mu \mathrm{l})$ was infused into the $\mathrm{mPFC}$ in a similar manner, or TTX $(15 \mathrm{ng} / 1.5 \mu \mathrm{l})$ was infused into the lateral PFC (from bregma, AP, 3.0; ML, 1.4; DV, 3.5). At the end of the experiment, the injection site was verified using standard histology methods.

Data analysis. All data analyses were performed in Microsoft (Redmond, WA) Excel using in-house programs written in Visual Basic for Applications. The onset of a burst was identified as two consecutive spikes with an interstimulus interval (ISI) $<80 \mathrm{~ms}$ and the termination of a burst was defined as an ISI $>160 \mathrm{~ms}$ (Grace and Bunney, 1984). Firing rate, bursting (number of spikes occurring in bursts), and the variability of firing were determined every $10 \mathrm{~s}$. The variability of firing was estimated by the coefficient of variation (CV), which equals the SD of ISIS divided by the mean (expressed as a percentage).

Firing periodicity was analyzed using methods similar to those described previously (Shi et al., 2004; Shi, 2005; Zhou et al., 2006). Briefly, rate histograms with a bin width of $50 \mathrm{~ms}$ were constructed based on 2 min recordings selected from each cell. After tapering using the Hanning-Tukey window function ( 15 windows with $50 \%$ overlap) and removal of the linear trend, a fast Fourier transform was performed to yield spectra with resolutions of $0.078 \mathrm{~Hz}$. The amplitude of a spectral peak is expressed as a percentage of total power so that the sum of all peaks equals 100. To help visualize slow changes, rate histograms were smoothed using methods described previously (Shi et al., 2004). All analyses, however, were based on nonsmoothed data. Autospectra of LFPs (down-sampled to $20 \mathrm{~Hz}$ ) were obtained using similar methods.

The degree of correlation between recordings was evaluated using cross-spectral and coherence analysis. Coherence was calculated from the cross-spectral density between the two recordings normalized by the spectral density of each signal. $y$-axis values in a coherence spectrum are squared coherence $\left(r^{2}\right)$, which ranges from 0 to 1 and is a measure of the stochastic coupling of two signals within a certain frequency band. Uncorrelated signals yield $r^{2}$ of 0 , whereas the more two signals are correlated, the more $r^{2}$ will increase toward 1. A 95\% confidence level was determined by $C=1-(1-\alpha)^{1 /(k-1)}$, where $\alpha=0.95$ and $k$ is the number of nonoverlapping windows (Rosenberg et al., 1989). Phase lags were calculated from portions of the phase spectrum encompassing the frequencies showing a significant coherence and a linear phase relationship between the signals. $y$-axis values in a phase spectrum are expressed as fractions of a cycle period. When two signals are in phase, the value is 0 , whereas if they are in antiphase (i.e., the first signal precedes or lags behind the second by $50 \%$ of a cycle period), the value is either 0.5 or -0.5 .

Differences between cell groups were determined by ANOVA followed by post hoc Tukey tests or by Kolmogorov-Smirnov tests. Effects of TTX were evaluated by comparing recordings before and after TTX infusion using paired $t$ tests. Spectral data were log-transformed before being subjected to statistical comparison. All values were based on analysis of 2 min recordings obtained under different conditions. All numerical results were expressed as mean \pm SEM.

\section{Results}

Covariation of DA cell firing with PFC LFPs

Based on the relative amplitude of the $\mathrm{SO}$, we previously divided VTA DA neurons into SO and non-SO cells (Shi, 2005). The term non-SO, however, is misleading because some non-SO cells also display an SO, although its amplitude is smaller than that of SO cells (Fig. 1C). To avoid confusion, this report will refer to SO and non-SO cells as high- (HS) and low-SO cells (LS), respectively. In 232 VTA DA cells recorded in this study, 47\% met the criterion for HS cells $(n=109)$ (Fig. $1 A, B)$. In the autospectra of these cells, the mean spectral density between 0.5 and $1.5 \mathrm{~Hz}\left(P_{0.5-1.5}\right.$ $\mathrm{Hz})$ was significantly higher than that between 0 and $3 \mathrm{~Hz}\left(P_{0-3}\right.$ $\mathrm{Hz}$ ) (Shi, 2005). As observed previously, these cells tended to fire spikes in clusters in a repetitive manner, making them appear to be rhythmic bursting cells. The remaining DA neurons were identified as LS cells because $P_{0.5-1.5} \mathrm{~Hz}$ was not significantly higher than $P_{0-3 \mathrm{~Hz}}$ (Fig. $1 C, D$ ).

To determine whether the SO is correlated with PFC cell activity, PFC LFPs were simultaneously recorded. In $67 \%$ of VTA DA neurons (156 of 232), the SO was significantly coherent with PFC LFPs, with a mean squared peak coherence $\left(r^{2}\right)$ of $0.66 \pm$ 0.01 . We referred to these DA neurons as PFC-coupled (PC) cells (Fig. $1 A, C$ ). In the remaining cells, no significant coherence was observed (peak $r^{2}<0.3123 ; n=47$ ), or the firing of the cell was correlated with PFC LFPs at frequencies $<0.5 \mathrm{~Hz}(n=4)$ or $>1.5$ $\mathrm{Hz}(n=25)$ and had a relatively low $r^{2}(0.38 \pm 0.01 ; n=29)$. For simplicity, these neurons were collectively referred to as non-PC cells (Fig. $1 B, D$ ).

\section{PC cells included both HS and LS cells}

The number of PC cells (156) was larger than the number of HS cells (109), suggesting that some LS cells are also functionally coupled to the PFC. Confirming this suggestion, 60 of 156 PC 
A DA Neuron (HS PC)
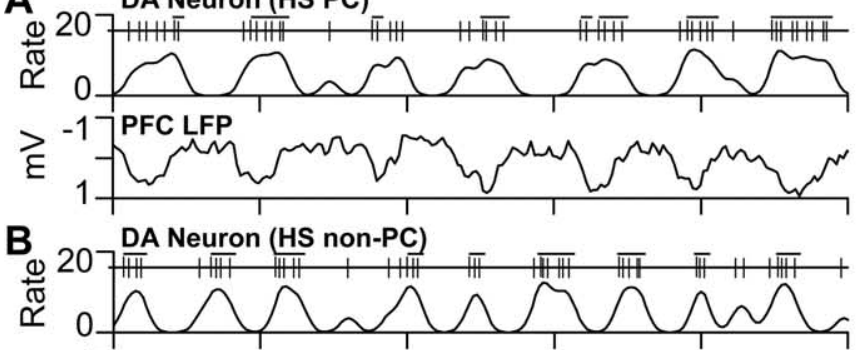

$z^{-.47}$ PFC LFP

C DA Neuron (LS PC)
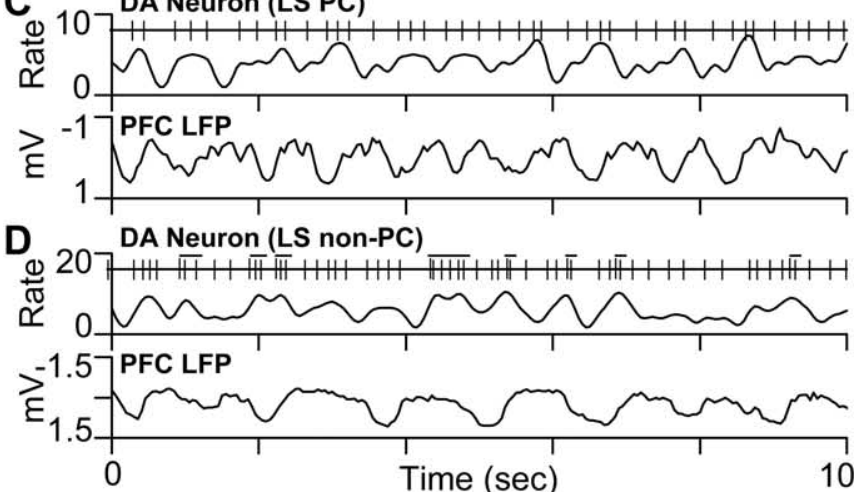

E

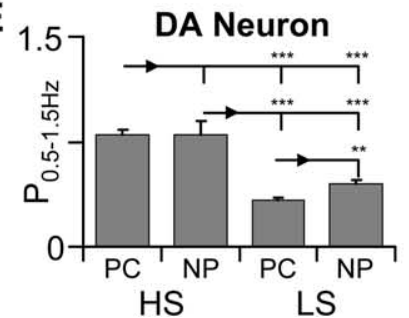

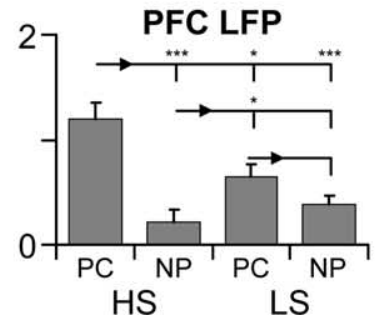
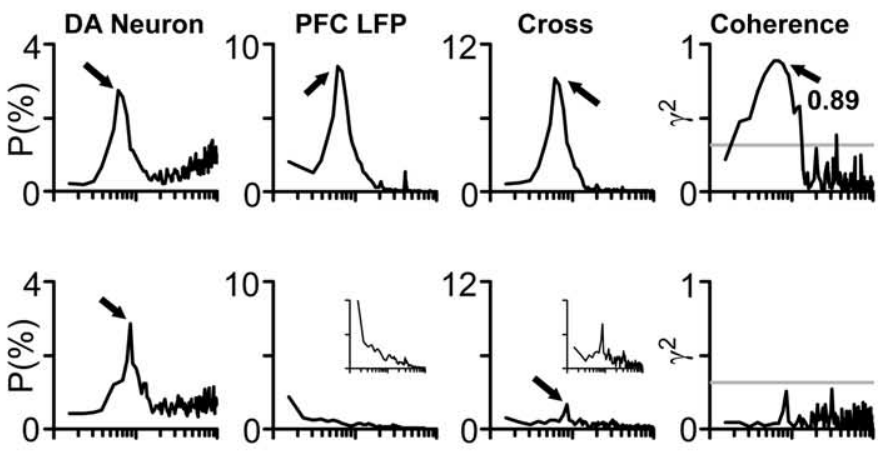
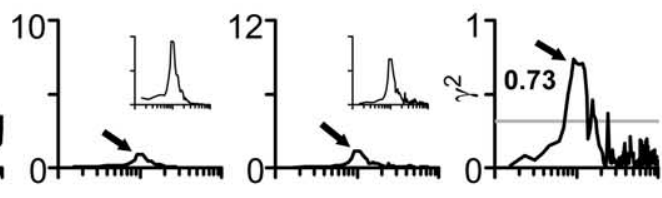

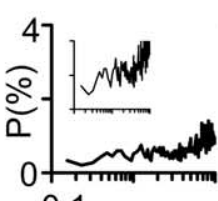

0.1

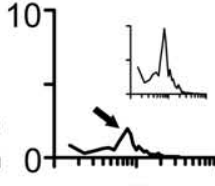

Frequency $(\mathrm{Hz})$
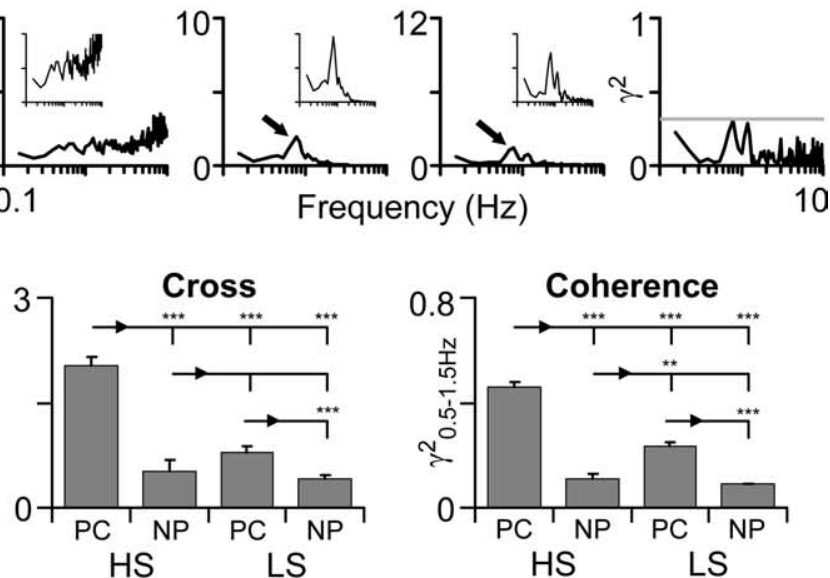

Figure 1. Correlation between firing patterns of VTA DA neurons and PFC LFPs. $A$, Left, Segments of spike trains recorded from an HS, PC DA neuron, the corresponding smoothed 50 ms bin-width rate histogram, and the simultaneously recorded PFC LFPs. Bursts, defined by the " $80 / 160$ ms" criterion, are marked by the horizontal bars above the spike train. Right, The two left charts are the autospectrum of a 2 min rate histogram from the same cell and the autospectrum of the concurrently recorded PFC LFPs, respectively. The two right charts are cross and coherence spectra between the two recordings. The horizontal shaded line in the coherence spectrum is the upper limit of a $95 \%$ confidence level. Both the firing of the cell and PFC LFPs displayed an SO (0.62 Hz; arrow). Cross and coherence spectra show that the two recordings were significantly correlated, with the peak squared coherence $r^{2}$ reaching 0.89 (arrow). In this and the following figures, LFP recordings are shown with the negative up. The amplitude of a spectral peak is expressed as a percentage of total power so that the sum of all peaks equals 100 . $\boldsymbol{B}$, Data from an HS non-PC neuron. The firing of this DA cell, but not the simultaneously recorded PFC LFPs, exhibited a clear SO (arrow). Cross and coherence analyses showed that the two recordings were not significantly correlated. The insets in the autospectra and cross spectra are the same spectra displayed on an expanded $y$-axis scale. $C$, Recordings from an LSPC cell. The cell was identified as an $L S$ cell because the mean power between 0.5 and $1.5 \mathrm{~Hz}\left(P_{0.5-1.5 \mathrm{~Hz}}\right)$ in its autospectrum was not significantly higher than that between 0 and $3 \mathrm{~Hz}\left(P_{0-3 \mathrm{~Hz}}\right)$. A small but clear spectral peak was visible at $\sim 0.94 \mathrm{~Hz}$ (arrow). At the same frequency, the concurrently recorded PFC LFPs also displayed a small SO. Cross and coherence analyses suggest that the two recordings are correlated $\left(r^{2}=0.73\right)$. $D$, Results from an $L S$ non-PC cell. The firing of this cell did not show a clear SO or a significant correlation with PFC LFPs, although the latter exhibited a clear SO (arrow). $\boldsymbol{E}$, Bar graphs showing differences between four groups of DA neurons (HSPC, HS non-PC, LSPC, and LS non-PC) in $P_{0.5-1.5 \mathrm{~Hz}}$ of autospectra and cross spectra and in the mean $r^{2}$ between 0.5 and $1.5 \mathrm{~Hz}\left(r^{2}{ }_{0.5-1.5 \mathrm{~Hz}}\right) . \mathrm{NP}, \mathrm{Non}-\mathrm{PC}$. ${ }^{*} p<0.05$; ${ }^{* *} p<0.01 ;{ }^{* * *} p<0.001$ (ANOVA and Tukey post hoc test).

cells were identified as LS cells. As expected, these cells showed relatively small spectral peaks between 0.5 and $1.5 \mathrm{~Hz}$ in their autospectra (Fig. 1C,E).

Most non-PC neurons were LS cells (63 of 76) (Fig. 1D). However, 13 non-PC cells were qualified as HS DA neurons (Fig. $1 B$ ). The magnitude of the $\mathrm{SO}$ in these cells was not significantly different from that in HS PC neurons (Fig. $1 E$ ). However, the SO in the concurrently recorded PFC LFPs, $P_{0.5-1.5 \mathrm{~Hz}}$ in cross spectra, and the mean $r^{2}$ between 0.5 and $1.5 \mathrm{~Hz}\left(r^{2}{ }_{0.5-1.5 \mathrm{~Hz}}\right)$ were all significantly smaller compared with HS PC cells (Fig. $1 B, E$ ).

Thus, based on the amplitude of the $\mathrm{SO}$ and its correlation with PFC LFPs, VTA DA neurons can be divided into four groups: HS PC $(41 \% ; n=96)$, LS PC $(26 \% ; n=60)$, HS non-PC
$(6 \% ; n=13)$, and LS non-PC $(27 \% ; n=63)$. Figure $1 E$ compares $P_{0.5-1.5 \mathrm{~Hz}}$ and $r^{2}{ }_{0.5-1.5 \mathrm{~Hz}}$ between these cells (ANOVA followed by post hoc Tukey's test). In the four groups of VTA DA neurons, HS PC cells exhibited the highest degree of coupling to PFC LFPs.

Role of the PFC in the SO of DA neurons

Two experiments were performed to further determine whether the SO in VTA DA neurons depends on PFC inputs. First, transections were made immediately caudal to the PFC ( $2 \mathrm{~mm}$ anterior to bregma) to mechanically interrupt PFC inputs to DA neurons. After the transection, both the number of DA neurons qualified as HS cells and the average amplitude of the SO were markedly decreased compared with nonlesioned rats. Thus, the 

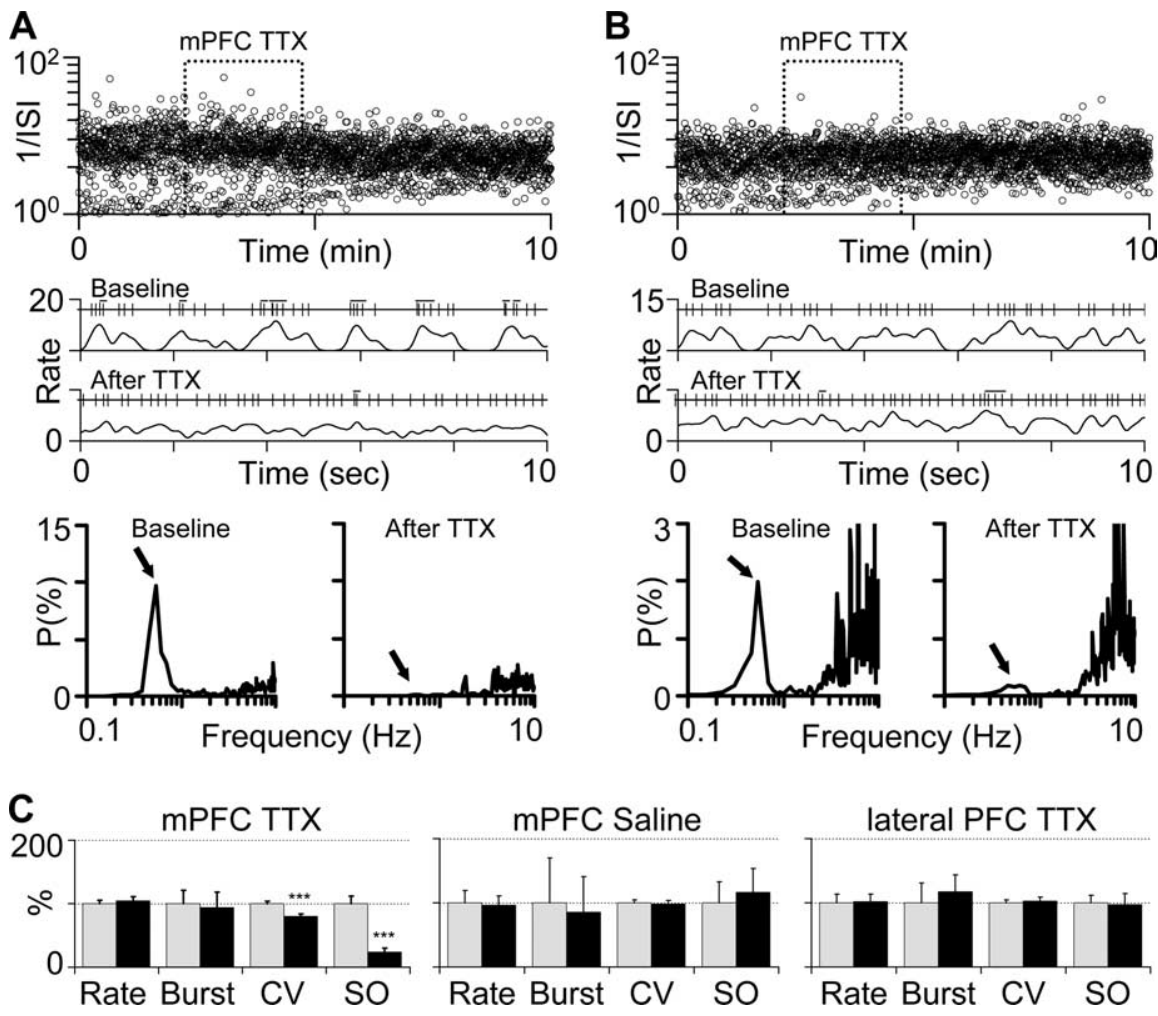

Figure 2. Effects of intra-mPFC infusion of TTX on DA neurons. $A$, Top, ISI plots of a VTA DA neuron showing that TTX infusion into the PFC changed ISI distribution from bimodal (two bands) to unimodal (one band). The top band seen during baseline corresponds to ISIs within the burst-like events and the bottom band corresponds to intervals between those events. Middle, Segments of spike trains and corresponding rate histograms obtained from the same cell before and after ITX infusion. Under baseline conditions, the cell fired spikes in clusters in a rhythmic manner. After TTX infusion, the cell exhibited a more regular firing pattern. PFC TTX infusion also decreased the number of spikes occurring in bursts defined by the "80/160 ms" criterion (marked by the horizontal bars above spike trains). Bottom, Power spectra from the same cell showing the presence of an SO (arrow) before TTX infusion and its inhibition after TTX infusion. $\boldsymbol{B}$, Results from a different VTA DA neuron showing that TTX infusion into the PFC also changed ISI distribution from bimodal to unimodal (top) and decreased the SO (middle and bottom). The infusion, however, increased the traditionally defined bursting (middle). C, Summary bar graphs showing effects of intra-mPFC TTX and saline and intralateral PFC TTX on VTA DA neurons. Shaded and solid bars represent values obtained before and after TTX infusion. All are expressed as percentage of baseline values. TTX infusion into the $\mathrm{MPFC}$ decreased CV and the S0. Intra-mPFC saline or intralateral PFC TTX produced no significant effects on all measures. ${ }^{* *} p<0.001$ (paired $t$ test).

percentage of cells qualified as HS cells was decreased from $47 \%$ in nonlesioned rats to only $17 \%$ in PFC-lesioned rats. The size of the SO, measured by $P_{0.5-1.5 \mathrm{~Hz}}$, was decreased from $0.43 \pm 0.04 \%$ $(n=232)$ in nonlesioned rats to $0.09 \pm 0.01 \%$ in lesioned rats ( $n=230 ; t=9.96 ; p<0.001, t$ test $)$.

In the second series of experiments, TTX (15 ng/1.5 $\mu \mathrm{l} / 2 \mathrm{~min})$ was locally infused to inactivate the ipsilateral medial PFC. In these experiments, only one cell was studied in each rat and recordings were selectively made from DA cells showing a clear SO. After a stable baseline recording, TTX was infused while the recording continued (Fig. $2 A, B$ ). Comparison of recordings before and after TTX infusion showed that mPFC inactivation decreased the SO in 25 of 27 cells tested and produced a small increase in the $\mathrm{SO}$ in the two remaining cells. On average, $P_{0.5-1.5 \mathrm{~Hz}}$ was decreased from $1.4 \pm 0.2$ to $0.5 \pm 0.1 \%(t=6.14 ; p<0.001$, paired $t$ test) (Fig. 2C).

TTX infusion also reduced the CV from $78.2 \pm 2.6$ to $62.1 \pm$ $3.5 \%(t=6.72 ; p<0.001)$ (Fig. 2C). Its effects on bursting and firing rate, however, were mixed. In 10 DA cells, PFC TTX infusion decreased the number of spikes occurring in bursts from an average of $1.18 \pm 0.29$ to $0.52 \pm 0.23$ spikes $/ \mathrm{s}(t=4.16 ; p<0.01)$ (Fig. $2 A)$. The effect was associated with no significant change in firing rate (from $4.43 \pm 2.3$ to $4.44 \pm 3.2$ spikes/s; $t=0.05 ; p=0.96)$. In eight DA cells, PFC inactivation increased the number of spikes occurring in bursts from $1.00 \pm 0.29$ to $1.65 \pm 0.44(t=3.66 ; p<$ 0.01 ) (Fig. $2 B$ ). This increase was accompanied by an increase in firing rate (from $5.48 \pm 0.49$ to $6.66 \pm 0.63 ; t=3.51 ; p=$ $0.01)$. In the remaining nine DA cells, the change in bursting induced by PFC inactivation was $<0.1$ spikes/s (from $0.11 \pm$ 0.05 to $0.12 \pm 0.07 ; t=0.12 ; p=0.91$ ). The firing rate of these cells was also not significantly changed by PFC inactivation (from $4.25 \pm 0.21$ to $3.67 \pm 0.18 ; t=1.4$; $p=0.20)$. When all cells were pooled, changes in both bursting (from $0.77 \pm$ 0.16 to $0.72 \pm 0.20 ; t=0.40 ; p=0.69)$ (Fig. $2 C$ ) and firing rate (from $3.82 \pm 0.20$ to $3.99 \pm 0.25 ; t=1.28$; $p=0.21$ ) (Fig. $2 C)$ were insignificant. Saline infusion in a similar manner produced no significant effects on all measures ( $n=5)$ (Fig. 2C). Infusion of TTX into the lateral PFC also produced no significant effects $(n=10)$ (Fig. 2C).

Phase analysis of PFC-DA cell coupling Phase analysis provides information on time delay between two synchronized signals. The analysis suggests that the SO in most DA neurons has a nearly antiphase relationship (i.e., inverse relationship) with PFC oscillations. As exemplified by the cell shown in Figure $3 A$ (see also Fig. $1 A, C)$, the firing of the cell coincided with positive deflections (i.e., hyperpolarization), rather than negative deflections in PFC LFPs. As in most other DA cells, the phase lag between the SO in this DA cell and that in the PFC was $>40 \%$ of a cycle period (Fig. $3 A, C$ ). A Kolmogorov-Smirnov test of the cumulative distributions of phase lags suggests that HS and LS cells are not significantly different regarding their phase relationships to the PFC $(d=0.16 ; p=0.32)$.

Relationship between PFC cell firing and PFC LFPs

To be certain that PFC LFPs reflect synchronous activity of PFC neurons and that negative deflections in PFC LFPs correspond to the active, up states of these cells, single-unit recordings were made from the PFC. Of all 122 cells recorded, the duration of the action potential was $>1.5 \mathrm{~ms}$, suggesting that they are pyramidal neurons (Tseng et al., 2006). In 71\% of cells (87 of 122), an SO similar to that seen in VTA DA neurons was observed. In 81 of 87 cells, the SO showed significant coherence with PFC LFPs, and in all 81 cells, the SO had a nearly in-phase relationship with PFC LFPs (Fig. $3 B, C$ ).

Relationship between DA cell firing and VTA LFPs

VTA LFPs have been shown to also exhibit an SO significantly correlated with PFC cell activity (Peters et al., 2004). To determine whether they reflect the activity of DA neurons, LFPs were recorded using the same electrodes used for single-unit recordings from DA neurons. In 145 of 232 pairs, a significant coher- 
ence was observed between the firing of the cell and LFPs (Fig. 4A). Most pairs (117 of 145), however, exhibited a nearly antiphase relationship with each other (|lag $>30 \%$ ) (Fig. 4B, left). VTA LFPs, however, showed a nearly in-phase relationship with PFC LFPs (Fig. 4A, B). On average, the SO in PFC LFPs led the SO in VTA LFPs by $2.0 \pm 0.4 \%$ of a cycle period, or $29.7 \mathrm{~ms}$ (Fig. $4 B$, right).

Relationship between the firing of VTA non-DA neurons and LFPs in the VTA and $P F C$

To determine whether firing patterns of VTA non-DA cells are also correlated with VTA LFPs, we made recordings from 153 VTA non-DA neurons. Of these cells, $30.7 \%$ showed significant coherence with VTA LFPs. This number is much smaller than that in DA neurons $(63 \% ; 145$ of 232 ). In non-DA neurons that showed coherence with VTA LFPs, the majority (64\%; 30 of 47) had a nearly in-phase relationship with the latter ( $|\operatorname{lag}|<20 \%$ ) (Fig. 5). In contrast, only $12 \%$ (18 of 145) of DA neurons that showed coherence with VTA LFPs exhibited similar properties (Fig. 5).

Firing patterns of non-DA neurons were also compared with simultaneously recorded PFC LFPs. Again, the percentage of non-DA cells that showed significant coherence with PFC LFPs was smaller than that observed in DA neurons (36 vs $67 \%)$. In this subset of non-DA cells, $62 \%$ (34 of 55) had a nearly in-phase relationship with PFC LFPs (Fig. 5). In DA neurons, only $13 \%$ (21 of 156) exhibited similar properties (Fig. 5).

\section{Discussion}

PFC stimulation has been shown to increase DA cell bursting and DA release (see Introduction). This study shows that, under nonstimulation conditions, the firing of most VTA DA neurons also covaries with PFC cell activity. In most of the cells, however, the firing rate, measured on a subsecond timescale, is inversely correlated with the degree of PFC depolarization. These results, together with previous findings, suggest that the PFC can exert both excitatory and inhibitory influences on VTA DA neurons.

Evidence for an inhibitory influence of the PFC on DA neurons

A correlation between DA cell firing and PFC LFPs was evident when rate histograms of DA neurons were directly compared with PFC LFPs. The correlation was further confirmed by cross-spectral and coherence analysis. To our surprise, nega-
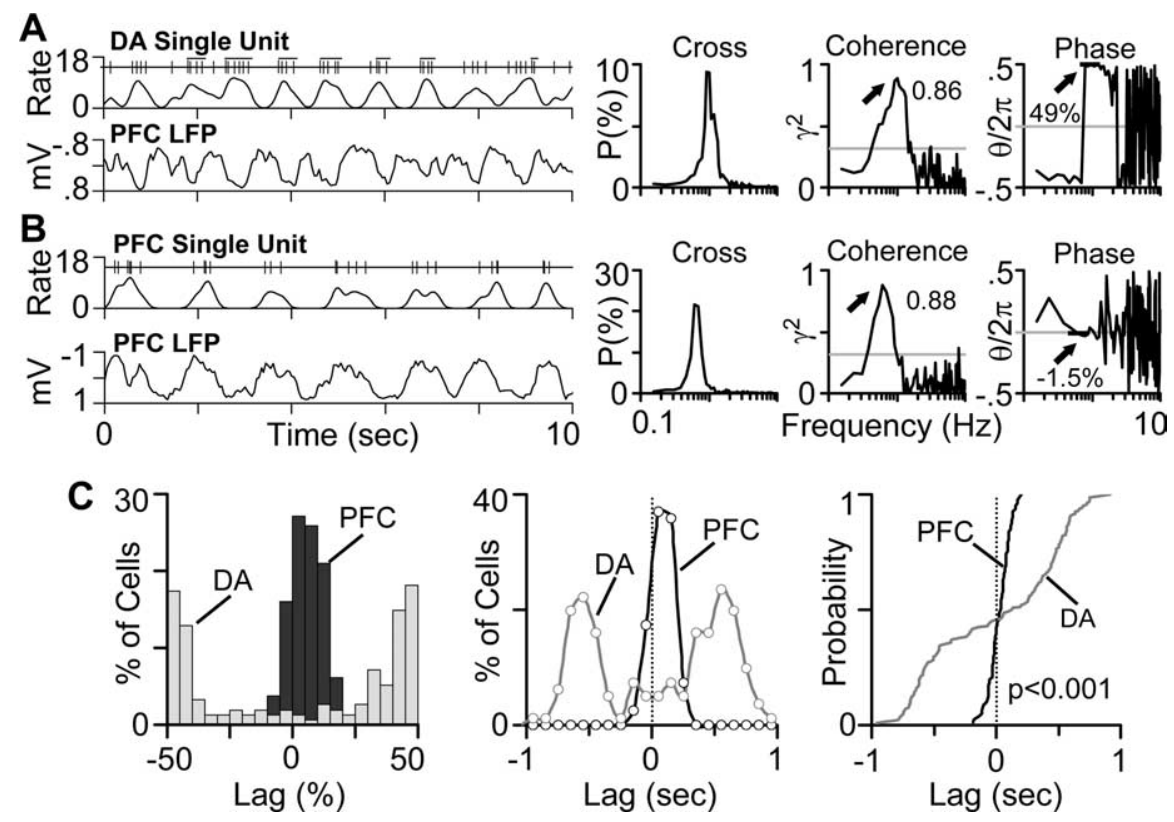

Figure 3. Phase relationship between DA cell firing and PFC LFPs. $\boldsymbol{A}$, Left, Segments of spike trains of a VTA DA neuron, the corresponding rate histogram, and PFC LFPs showing that the firing of the cell coincided with positive (downward) deflections in PFC LFPs. Right, Cross, coherence, and phase spectra showing that the $S 0$ in this DA cell preceded the $S 0$ in PFC LFPs by almost one half of a cycle period (49\%; arrow). $y$-axis values in the phase spectrum are fractions of an oscillation cycle. $\boldsymbol{B}$, Left, Segments of spike trains recorded from a PFC neuron, the corresponding rate histogram, and PFC LFPs recorded via the same electrode. Right, Cross, coherence, and phase spectra showing that the SO in this PFC neuron was nearly in-phase with respect to the SO in PFC LFPS $(-1.5 \%$ of a cycle period). C, Left, Distributions of phase lags between DA cell firing and PFC LFPs (shaded bars) and between PFC cell firing and PFC LFPs (solid bars). The SO in most VTA DA neurons preceded or lagged behind the SO in PFC LFPs by $>40 \%$ of an oscillation cycle. In contrast, phase lags between the $S 0$ of PFC neurons and that in PFC LFPs were all $<20 \%$ of a cycle period. Values on the $y$-axis are percentages of cells that showed significant coherence with PFC LFPs. Center, Distributions of the same phase lags expressed in time (sec). Right, Cumulative distributions of phase lags. A Kolmogorov-Smirnov test suggests that DA and PFC neurons are significantly different in terms of their phase relationship to PFC LFPs.
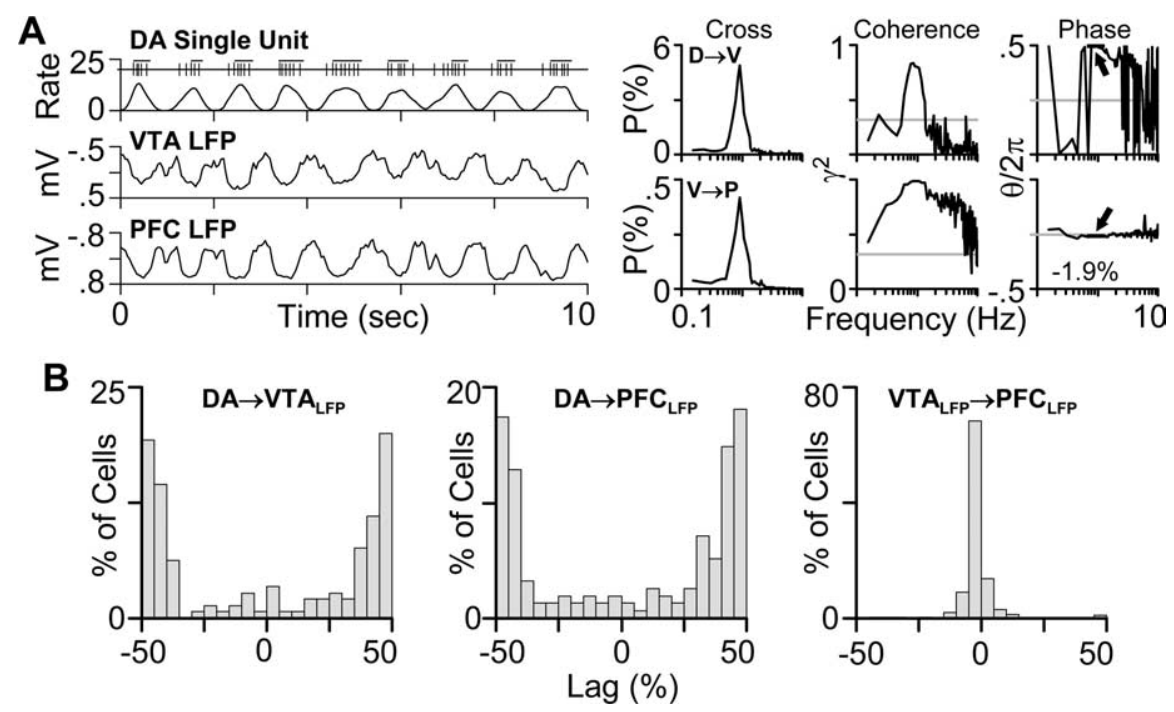

Figure 4. Phase relationship between DA cell firing and VTA LFPs. $\boldsymbol{A}$, Left, Segments of spike trains recorded from a VTA DA neuron, the corresponding rate histogram, and concurrently recorded LFPs from the VTA and PFC. Right, Cross, coherence, and phase spectra between the firing of the DA cell and VTA LFPs ( $D \rightarrow V$, top charts) and between VTA and PFC LFPs (V $\rightarrow P$, bottom charts). As in most DA neurons, the SO in this DA cell had a nearly antiphase relationship with VTA LFPs. The latter showed a nearly in-phase relationship with PFC LFPs. $\boldsymbol{B}$, Distributions of phase lags between DA cell firing and VTA LFPs (DA $\rightarrow V_{\text {LFP, }}$ i left), between $D A$ cell firing and PFC LFPs (DA $\rightarrow P_{L F P}$; center), and between VTA and PFC LFPs (V $V_{L F P} \rightarrow P_{L F P}$; right). $y$-axis values are percentages of cells that showed significant coherence with PFC or VTA LFPs 

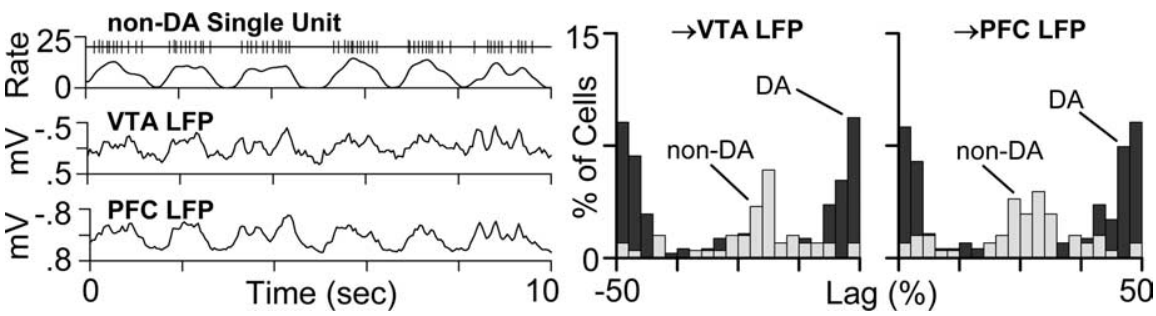

Figure 5. Phase relationship between the firing of VTA non-DA neurons and LFPs in the VTA and PFC. Left, Segments of spike trains from a VTA non-DA neuron, the corresponding rate histogram, and concurrently recorded VTA and PFC LFPs. Unlike most DA neurons, this non-DA cell fired in synchrony with negative deflections in both VTA and PFC LFPs. Right, Distributions of phase lags between the SO of VTA non-DA neurons and that in VTA LFPs (left chart; shaded bars) and PFC LFPs (right chart; shaded bars). For comparison, data from DA neurons are also shown as solid bars. Values on the $y$-axis are percentages of all DA or non-DA cells recorded, including those that did not show significant coherence with VTA or PFC LFPs.

tive deflections in PFC LFPs coincided with decreases, instead of increases, in firing in most VTA DA neurons examined. As shown in the present and previous studies (Destexhe et al., 1999; Haider et al., 2006; Mukovski et al., 2006), negative deflections in PFC LFPs correspond to the active, up states of PFC neurons. These findings and the fact that the SO in DA neurons is inhibited by PFC lesions or inactivation suggest that PFC information is transferred to DA neurons at least partially through inhibitory relay neurons.

Consistent with the above suggestion, most VTA DA neurons, including those projecting to the NAc, do not receive direct inputs from the PFC (Carr and Sesack, 2000a; Sesack et al., 2003). PFC terminals, however, form direct contacts with some VTA GABA neurons, raising the possibility that part of PFC influence on DA neurons is indirectly mediated through these inhibitory cells. Consistent with this possibility, the $\mathrm{GABA}_{\mathrm{A}}$ agonist muscimol, which has been shown to preferentially inhibit GABA neurons in the VTA and the substantia nigra (MacNeil et al., 1978; Walters and Lakoski, 1978; Grace and Bunney, 1979; Waszczak et al., 1980; Waszczak and Walters, 1980; Tepper et al., 1995), inhibits the SO in DA neurons (Shi et al., 2004). Also consistent with this possibility, a subset of VTA non-DA neurons was found to fire in synchrony with the active, up states of PFC cells. In addition to a possible involvement of VTA GABA neurons, other inhibitory nuclei such as the NAc may also play a role in relaying PFC information to DA neurons. Studies have shown that the NAc receives direct excitatory inputs from the PFC (Sesack and Pickel, 1992; Brady and O'Donnell, 2004) and provides inhibitory projections to DA neurons (Phillipson, 1979; Maeda and Mogenson, 1980; Yim and Mogenson, 1980).

An inhibitory influence of the PFC on DA neurons is also suggested by the previous finding that the bursts in DA neurons induced by PFC stimulation are often preceded by an inhibition, which lasts up to several hundred milliseconds (Nakamura et al., 1979; Thierry et al., 1979; Gariano and Groves, 1988; Overton et al., 1996; Tong et al., 1996; Overton and Clark, 1997). According to Tong et al. (1996), PFC stimulation produces an inhibition or an initial inhibition followed by an excitation in $67 \%$ of DA cells tested. In 29\%, the stimulation induces an excitation followed by an inhibition. In only $3 \%$, PFC stimulation induces excitation only.

\section{Evidence for a dual influence of the PFC on DA neurons}

Muscimol not only inhibits the SO, but also increases the firing rate of VTA DA neurons (Shi et al., 2004). PFC lesions or inactivation, however, inhibited the $\mathrm{SO}$ without significantly altering the firing rate. One possible explanation is that the PFC exerts both inhibitory and excitatory influences on DA neurons. Muscimol increases DA cell firing by selectively removing the inhibitory influence mediated through VTA GABA neurons. PFC lesions or inactivation, however, remove both excitatory and inhibitory influences and, thus, produce no significant effect on firing rate. The above discussed biphasic response of DA neurons to PFC stimulation also supports a dual influence of the PFC on DA neurons. It is interesting to note that the duration of the biphasic response is nearly identical to that of the SO $(\sim 1 s)$, raising the possibility that pathways underlying the response are also involved in generating the SO. With the data available, it is still unclear how different pathways interplay to cooperatively generate the SO in DA neurons. Results with muscimol suggest that inhibitory pathways, by rhythmically inhibiting DA neurons, may carry the frequency information of the SO, whereas excitatory pathways may provide a relatively tonic excitation and determine the amplitude of the SO.

\section{Direction of coupling between PFC and DA neurons}

The SO in DA neurons and that in the PFC may be mutually dependent because some VTA DA neurons project back to the PFC (Thierry et al., 1973; Berger et al., 1976; Lindvall et al., 1978). Non-PFC projecting DA neurons may also influence the PFC indirectly through their projections to other brain areas. Consistent with this possibility, inactivation of the VTA by local infusion of lidocaine inhibits the SO in PFC neurons (Peters et al., 2004). Additional experiments, however, are needed to rule out the possibility that the effect of lidocaine is attributable to its inactivation of VTA non-DA neurons, some of which are known to project to the PFC (Carr and Sesack, 2000b), or to inactivation of fibers passing through the VTA.

Phase analysis may help further determine the direction of coupling between two synchronized signals. However, because of the discrete nature of single-unit recording, the analysis in the present study provided only a rough estimate of the phase relationship between DA cells and the PFC. Paired LFP recordings would provide a better measurement if VTA LFPs reflected the activity of DA neurons. In this study, we found that only $8 \%$ of all DA neurons (18 of 232) and 20\% (30 of 153) of all non-DA neurons showed a nearly in-phase relationship with VTA LFPs, suggesting that VTA LFPs reflect the activity of only a small subset of VTA DA and non-DA neurons. However, because many more DA neurons (117 of 232) had a nearly antiphase relationship with VTA LFPs, it is possible that because of the morphology and anatomical arrangement of DA neurons in the VTA, negative deflections in VTA LFPs reflect synchronized hyperpolarization, instead of depolarization, of DA neurons. If this proves to be correct, paired VTA-PFC LFP recordings would suggest that the up states of PFC neurons precede the hyperpolarization in most PFC-coupled DA neurons by $\sim 30 \mathrm{~ms}$.

Variability of coupling between PFC and DA neurons Although most PFC-coupled DA neurons had a nearly antiphase relationship with the PFC, a few exhibited a nearly in-phase relationship. The SO in this group of cells may be derived monosynaptically from the PFC because some VTA DA neurons do receive direct inputs from the PFC (Carr and Sesack, 2000a; Sesack et al., 
2003). In 33\% of DA neurons, no significant coherence was found between the firing of the cell and PFC LFPs, suggesting a lack of innervation of these neurons by inputs derived from the recording site in the PFC or that, during the time of recording, the pattern of activity in the PFC does not favor information transmission from the PFC to DA neurons. Consistent with this latter possibility, PFC LFPs usually exhibited a small or no significant SO during the time of recording from a non-PFC coupled DA cell, and most non-PFC coupled cells were low-SO cells. Thus, the SO may be more favorably transferred from the PFC to VTA DA neurons than oscillatory signals of other frequencies, and some non-PFC-coupled DA neurons may show significant coupling to the PFC when the SO in the PFC is increased. Previous evidence suggests that psychostimulants including D-amphetamine and cocaine enhance the SO in DA neurons by increasing norepinephrine release and activation of $\alpha 1$ receptors in the PFC (Darracq et al., 1998; Ventura et al., 2003; Shi et al., 2004, 2007; Zhou et al., 2006). A few non-PFC-coupled DA cells, however, showed a significant $\mathrm{SO}$, whereas the concurrently recorded PFC LFPs did not. This observation and the finding that PFC lesions and inactivation inhibit the SO in most, but not all, DA neurons suggest that, in some DA cells, the SO can be generated independent of a PFC influence.

In summary, this study shows that in most VTA DA neurons, the previously described SO (rhythmic bursting) encodes information from the PFC. In many cells, however, the SO is reversed relative to PFC activity, suggesting that part of PFC information is transferred to DA neurons through inhibitory relay neurons. The inverse correlation also raises the possibility that action potential-dependent DA release from DA terminals and glutamate release from PFC terminals are coordinated on a subsecond timescale such that when glutamate release is high, DA release is low. Additional understanding of this coordinated activity may provide critical new insights into disorders thought to involve both VTA DA and PFC neurons, including schizophrenia, drug addiction, and attention deficit hyperactivity disorder.

\section{References}

Berger B, Thierry AM, Tassin JP, Moyne MA (1976) Dopaminergic innervation of the rat prefrontal cortex: a fluorescence histochemical study. Brain Res 106:133-145.

Brady AM, O'Donnell P (2004) Dopaminergic modulation of prefrontal cortical input to nucleus accumbens neurons in vivo. J Neurosci 24:1040-1049.

Bunney BS, Grace AA (1978) Acute and chronic haloperidol treatment: comparison of effects on nigral dopaminergic cell activity. Life Sci 23:1715-1727.

Bunney BS, Walters JR, Roth RH, Aghajanian GK (1973) Dopaminergic neurons: effect of antipsychotic drugs and amphetamine on single cell activity. J Pharmacol Exp Ther 185:560-571.

Carr DB, Sesack SR (2000a) Projections from the rat prefrontal cortex to the ventral tegmental area: target specificity in the synaptic associations with mesoaccumbens and mesocortical neurons. J Neurosci 20:3864-3873.

Carr DB, Sesack SR (2000b) GABA-containing neurons in the rat ventral tegmental area project to the prefrontal cortex. Synapse 38:114-123.

Chiodo L, Bunney B (1983) Typical and atypical neuroleptics: differential effects of chronic administration on the activity of A9 and A10 midbrain dopaminergic neurons. J Neurosci 3:1607-1619.

Darracq L, Blanc G, Glowinski J, Tassin JP (1998) Importance of the noradrenaline-dopamine coupling in the locomotor activating effects of D-amphetamine. J Neurosci 18:2729-2739.

Destexhe A, Contreras D, Steriade M (1999) Spatiotemporal analysis of local field potentials and unit discharges in cat cerebral cortex during natural wake and sleep states. J Neurosci 19:4595-4608.

Gariano RF, Groves PM (1988) Burst firing induced in midbrain dopamine neurons by stimulation of the medial prefrontal and anterior cingulate cortices. Brain Res 462:194-198.
Gonon FG (1988) Nonlinear relationship between impulse flow and dopamine released by rat midbrain dopaminergic neurons as studied by in vivo electrochemistry. Neuroscience 24:19-28.

Grace AA, Bunney BS (1979) Paradoxical GABA excitation of nigral dopaminergic cells: indirect mediation through reticulata inhibitory neurons. Eur J Pharmacol 59:211-218.

Grace AA, Bunney BS (1983) Intracellular and extracellular electrophysiology of nigral dopaminergic neurons-1. Identification and characterization. Neuroscience 10:301-315.

Grace AA, Bunney BS (1984) The control of firing pattern in nigral dopamine neurons: burst firing. J Neurosci 4:2877-2890.

Haider B, Duque A, Hasenstaub AR, McCormick DA (2006) Neocortical network activity in vivo is generated through a dynamic balance of excitation and inhibition. J Neurosci 26:4535-4545.

Karreman M, Moghaddam B (1996) The prefrontal cortex regulates the basal release of dopamine in the limbic striatum: an effect mediated by ventral tegmental area. J Neurochem 66:589-598.

Lindvall O, Bjorklund A, Divac I (1978) Organization of catecholamine neurons projecting to the frontal cortex in the rat. Brain Res 142:1-24.

MacNeil D, Gower M, Szymanska I (1978) Response of dopamine neurons in substantia nigra to muscimol. Brain Res 154:401-403.

Maeda H, Mogenson GJ (1980) An electrophysiological study of inputs to neurons of the ventral tegmental area from the nucleus accumbens and medial preoptic-anterior hypothalamic areas. Brain Res 197:365-377.

Miller EK, Cohen JD (2001) An integrative theory of prefrontal cortex function. Annu Rev Neurosci 24:167-202.

Miller EK, Freedman DJ, Wallis JD (2002) The prefrontal cortex: categories, concepts and cognition. Philos Trans $\mathrm{R}$ Soc Lond B Biol Sci 357:1123-1136.

Mukovski M, Chauvette S, Timofeev I, Volgushev M (2006) Detection of active and silent states in neocortical neurons from the field potential signal during slow-wave sleep. Cereb Cortex, in press.

Murase S, Grenhoff J, Chouvet G, Gonon FG, Svensson TH (1993) Prefrontal cortex regulates burst firing and transmitter release in rat mesolimbic dopamine neurons studied in vivo. Neurosci Lett 157:53-56.

Nakamura S, Iwatsubo K, Tsai CT, Iwama K (1979) Cortically induced inhibition of neurons of rat substantia nigra (pars compacta). Jpn J Physiol 29:353-357.

Overton PG, Clark D (1997) Burst firing in midbrain dopaminergic neurons. Brain Res Brain Res Rev 25:312-334.

Overton PG, Tong ZY, Clark D (1996) A pharmacological analysis of the burst events induced in midbrain dopaminergic neurons by electrical stimulation of the prefrontal cortex in the rat. J Neural Transm 103:523-540.

Peters Y, Barnhardt NE, O’Donnell P (2004) Prefrontal cortical up states are synchronized with ventral tegmental area activity. Synapse 52:143-152.

Phillipson OT (1979) Afferent projections to the ventral tegmental area of Tsai and interfascicular nucleus: a horseradish peroxidase study in the rat. J Comp Neurol 187:117-143.

Rosenberg JR, Amjad AM, Breeze P, Brillinger DR, Halliday DM (1989) The Fourier approach to the identification of functional coupling between neuronal spike trains. Prog Biophys Mol Biol 53:1-31.

Sesack SR, Pickel VM (1992) Prefrontal cortical efferents in the rat synapse on unlabeled neuronal targets of catecholamine terminals in the nucleus accumbens septi and on dopamine neurons in the ventral tegmental area. J Comp Neurol 320:145-160.

Sesack SR, Carr DB, Omelchenko N, Pinto A (2003) Anatomical substrates for glutamate-dopamine interactions: evidence for specificity of connections and extrasynaptic actions. Ann NY Acad Sci 1003:36-52.

Shi WX (2005) Slow oscillatory firing: a major firing pattern of dopamine neurons in the ventral tegmental area. J Neurophysiol 94:3516-3522.

Shi WX, Pun CL, Zhang XX, Jones MD, Bunney BS (2000) Dual effects of $\mathrm{D}$-amphetamine on dopamine neurons mediated by dopamine and nondopamine receptors. J Neurosci 20:3504-3511.

Shi WX, Pun CL, Zhou Y (2004) Psychostimulants induce low-frequency oscillations in the firing activity of dopamine neurons. Neuropsychopharmacology 29:2160-2167.

Shi WX, Zhang XY, Pun CL, Bunney BS (2007) Clozapine blocks $\mathrm{D}$-amphetamine-induced excitation of dopamine neurons in the ventral tegmental area. Neuropsychopharmacology, in press.

Svensson TH, Tung CS (1989) Local cooling of pre-frontal cortex induces 
pacemaker-like firing of dopamine neurons in rat ventral tegmental area in vivo. Acta Physiol Scand 136:135-136.

Taber M, Fibiger H (1995) Electrical stimulation of the prefrontal cortex increases dopamine release in the nucleus accumbens of the rat: modulation by metabotropic glutamate receptors. J Neurosci 15:3896-3904.

Taber MT, Das S, Fibiger HC (1995) Cortical regulation of subcortical dopamine release: mediation via the ventral tegmental area. J Neurochem 65:1407-1410.

Tepper J, Martin L, Anderson D (1995) GABAA receptor-mediated inhibition of rat substantia nigra dopaminergic neurons by pars reticulata projection neurons. J Neurosci 15:3092-3103.

Thierry AM, Blanc G, Sobel A, Stinus L, Golwinski J (1973) Dopaminergic terminals in the rat cortex. Science 182:499-501.

Thierry AM, Deniau JM, Feger J (1979) Effects of stimulation of the frontal cortex on identified output VMT cells in the rat. Neurosci Lett 15:103-107.

Tong ZY, Overton PG, Clark D (1996) Stimulation of the prefrontal cortex in the rat induces patterns of activity in midbrain dopaminergic neurons which resemble natural burst events. Synapse 22:195-208.

Tseng KY, Mallet N, Toreson KL, Le Moine C, Gonon F, O’Donnell P (2006) Excitatory response of prefrontal cortical fast-spiking interneurons to ventral tegmental area stimulation in vivo. Synapse 59:412-417.
Ungless MA, Magill PJ, Bolam JP (2004) Uniform inhibition of dopamine neurons in the ventral tegmental area by aversive stimuli. Science 303:2040-2042.

Ventura R, Cabib S, Alcaro A, Orsini C, Puglisi-Allegra S (2003) Norepinephrine in the prefrontal cortex is critical for amphetamine-induced reward and mesoaccumbens dopamine release. J Neurosci 23:1879-1885.

Walters JR, Lakoski JM (1978) Effect of muscimol on single unit activity of substantia nigra dopamine neurons. Eur J Pharmacol 47:469-471.

Waszczak BL, Walters JR (1980) Intravenous GABA agonist administration stimulates firing of A10 dopaminergic neurons. Eur J Pharmacol 66:141-144.

Waszczak BL, Eng N, Walters JR (1980) Effects of muscimol and picrotoxin on single unit activity of substantia nigra neurons. Brain Res 188:185-197.

Yim CY, Mogenson GJ (1980) Electrophysiological studies of neurons in the ventral tegmental area of tsai. Brain Res 181:301-313.

You ZB, Tzschentke TM, Brodin E, Wise RA (1998) Electrical stimulation of the prefrontal cortex increases cholecystokinin, glutamate, and dopamine release in the nucleus accumbens: an in vivo microdialysis study in freely moving rats. J Neurosci 18:6492-6500.

Zhou Y, Bunney BS, Shi WX (2006) Differential effects of cocaine on firing rate and pattern of dopamine neurons: role of $\alpha 1$ receptors and comparison with L-dopa and apomorphine. J Pharmacol Exp Ther 317:196-201. 